\title{
Can Physical Tools that Adapt their Shape based on a Learner's Performance Help in Motor Skill Training?
}

\author{
Dishita Turakhia \\ dishita@mit.edu \\ CSAIL, MIT, Cambridge, MA, USA
}

\author{
Yini Qi \\ qyn@mit.edu
}

CSAIL, MIT, Cambridge, MA, USA

\author{
Lotta-Gili Blumberg \\ blumberg@mit.edu
}

CSAIL, MIT, Cambridge, MA, USA

\author{
Andrew Wong \\ andrewwo@mit.edu \\ CSAIL, MIT, Cambridge, MA, USA
}

\author{
Stefanie Mueller \\ stefanie.mueller@mit.edu \\ CSAIL, MIT, Cambridge, MA, USA
}
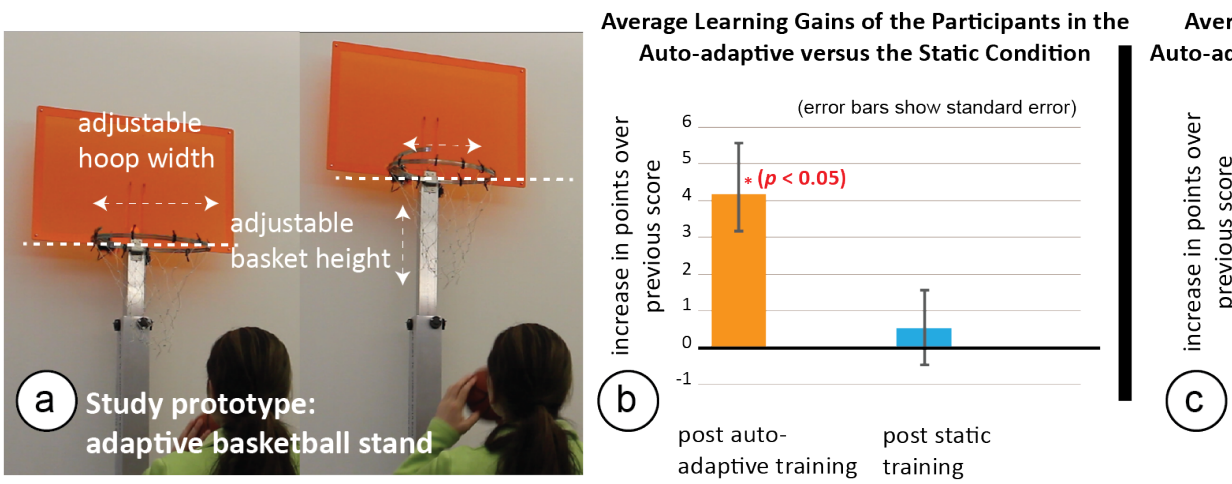

Average Learning Gains of the Participants in the Auto-adaptive versus the Manually Adaptive Condition

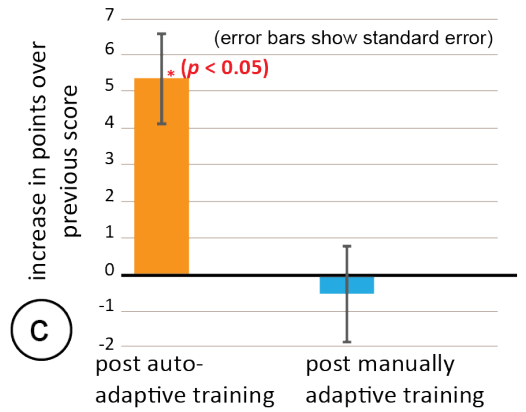

Figure 1: We investigate if adaptive learning tools that automatically adapt their shape to adjust the task difficulty based on a learner's performance can help in motor-skill training. To this end, we built (a) a study prototype in the form of an adaptive basketball stand that can adjust its hoop size and basket height. Our studies show that when the tool adapts automatically, training leads to significantly higher learning gains in comparison to training with (b) a static tool and (c) a manually adaptive tool for which the learners choose the difficulty level themselves.

\begin{abstract}
Adaptive tools that can change their shape to support users with motor tasks have been used in a variety of applications, such as to improve ergonomics and support muscle memory. In this paper, we investigate whether shape-adapting tools can also help in motor skill training. In contrast to static training tools that maintain task difficulty at a fixed level during training, shape-adapting tools can vary task difficulty and thus keep learners' training at the optimal challenge point, where the task is neither too easy, nor too difficult.

To investigate whether shape adaptation helps in motor skill training, we built a study prototype in the form of an adaptive basketball stand that works in three conditions: (1) static, (2) manually adaptive, and (3) auto-adaptive. For the auto-adaptive condition, the tool adapts to train learners at the optimal challenge point where the task is neither too easy nor too difficult. Results from our two user studies show that training in the auto-adaptive condition leads
\end{abstract}

This work is licensed under a Creative Commons Attribution International 4.0 License.

For all other uses, contact the owner/author(s)

TEI '21, February 14-17, 2021, Salzburg, Austria

ACM ISBN 978-1-4503-8213-7/21/02.

https://doi.org/10.1145/3430524.3440636 to statistically significant learning gains when compared to the static $\left(F_{1,11}=1.856, p<0.05\right)$ and manually adaptive conditions $\left(F_{1,11}=2.386, p<0.05\right)$.

\section{CCS CONCEPTS}

- Human-centered computing $\rightarrow$ User studies.

\section{KEYWORDS}

adaptive physical interfaces; motor skill training; tangible interfaces for learning

\section{ACM Reference Format:}

Dishita Turakhia, Yini Qi, Lotta-Gili Blumberg, Andrew Wong, and Stefanie Mueller. 2021. Can Physical Tools that Adapt their Shape based on a Learner's Performance Help in Motor Skill Training?. In Fifteenth International Conference on Tangible, Embedded, and Embodied Interaction (TEI '21), February 14-17, 2021, Salzburg, Austria. ACM, New York, NY, USA, 12 pages. https://doi.org/10.1145/3430524.3440636

\section{INTRODUCTION}

Research in HCI has shown that shape-adapting tools can support users in the execution of motor tasks by either helping users to perform the task correctly or by preventing users from making mistakes. For instance, actuated office furniture, such as a desk, chair, and monitor that adapt their position based on a user's body 
posture, can help users improve ergonomics [6, 34]. Similarly, actuated kitchenware, such as an actuated knife, can prevent users from getting injured by automatically retracting the blade if the knife gets too close to the user's fingers [37]. While these systems show that shape-adaptation can help users with the execution of motor tasks, it is unclear if such automatically shape-adapting tools can also help train users for the underlying motor skills so that after fully acquiring the skill, users no longer need additional support when performing the motor task.

In our work, we investigate if physical tools that automatically adapt their shape can help users in motor skill training. In particular, we study how training with an automatically adaptive training tool compares against the current methods of training, which either use static or manually adaptive tools. Static training tools, such as a pair of fixed-height training wheels for learning how to ride a bike, do not allow the user to adjust the difficulty of the training. Manually adaptive training tools, such as height-adjustable training wheels, do allow to vary the difficulty level, but users have to first assess their own skill level to determine which difficulty setting leads to optimal training. In contrast, automatically adaptive training tools that can sense a user's performance and then adapt the difficulty level accordingly, have the potential to allow users to always train at what is called the optimal challenge point, where the task is neither too easy, nor too difficult for their skill level.

To study if automatically adaptive tools can indeed lead to larger learning gains, we built a study prototype in the form of a basketball stand with adjustable hoop height and width that works under three training conditions: (1) static, (2) manually adaptive, and (3) auto-adaptive condition. For the static training condition, the basketball stand is at a fixed hoop height and width, and thus the difficulty level does not change over time. For the manually adaptive training condition, users can adjust the difficulty level themselves by controlling the hoop height and width by activating the actuators (motors) on the stand. For the auto-adaptive training condition, the tool automatically varies the difficulty level by adjusting the hoop height and width based on the user's performance that is detected using sensors, such as a switch on the net and a piezo sensor on the board.

To determine when to adapt the study prototype in the autoadaptive training condition, we use an adaptation algorithm that maintains the training difficulty at the optimal challenge point, at which the task is neither too difficult nor too easy for the users. We use the model of the optimal challenge point because studies in motor skill learning have shown that when coaches train learners at the optimal challenge point, learners have the maximum potential learning benefit [14]. To maintain the difficulty level at the optimal challenge point, the algorithm measures a users' performance and based on the performance over time, determines whether the tool should adapt to a more difficult setting, a less difficult setting, or remain at the current difficulty setting during the training.

To test if training in the auto-adaptive condition, in which users train at the optimal challenge point, leads to higher learning gains in motor skill training when compared to the static and manually adaptive condition, we conducted two user studies with 12 participants each. In the user study, we trained participants on the task of throwing a basketball into the hoop. We measured the participants' learning gains (i.e., increase in performance scores) after (1) training on the automatically adaptive tool versus the static training tool, and (2) training on the tool versus the manually adaptive training tool. Our study results show significant learning gains in the auto-adaptive training condition when compared to the static $\left(F_{1,11}=1.856, p<0.05\right)$ and manually adaptive conditions $\left(F_{1,11}=2.386, p<0.05\right)$. While we expected that the learning gains in the manually adaptive condition would also be substantial, we found that there was a mismatch between participants' skill levels and their own assessment of what task difficulty is best to train on, resulting in only small learning gains.

In addition to leading to higher learning gains, users also preferred training with auto-adaptive tools over training with static and manually adaptive tools. For instance, in user study 1, p11 said, 'adaptive training makes each stage of the training experience more rewarding, so it helped me focus better', and in user study 2, p3 said, 'auto-adaptive was easier to use; I didn't have to think about if I set it too easy or too hard.'. Thus, based on the results of our user studies, we see a large potential for the use of automatically adaptive training tools in motor skill training, making personalized training accessible to a larger audience that may not have access to an expert trainer.

In summary, we contribute:

- A study prototype of an adaptive basketball hoop that enables training in three conditions: (1) static, (2) manually adaptive, and (3) auto-adaptive condition. In auto-adaptive condition, the physical tool automatically adapts to vary the task difficulty based on the learner's performance, so that the task difficulty is at the optimal challenge point for the learner.

- A study with 12 participants measuring the learning gains of training in the auto-adaptive training condition versus the static training condition with results showing significantly higher learning gains in the auto-adaptive condition $\left(F_{1,11}=\right.$ $1.856, p<0.05)$.

- A study with 12 participants measuring the learning gains of training in the auto-adaptive training condition versus the manually adaptive training condition with results showing significantly higher learning gains in the auto-adaptive condition $\left(F_{1,11}=2.386, p<0.05\right)$.

\section{RELATED WORK}

Our work is related to (1) actuated shape-changing tools that support users in executing motor tasks, (2) current motor skill training systems that mainly provide multimodal feedback, and (3) motor skill learning approaches that vary task difficulty during training.

\subsection{Actuated Shape-changing Tools that Support Users in Executing Motor Tasks}

HCI researchers have developed several tools that use actuation to assist users in the correct execution of motor tasks [3, 27]. For example, Shin et al.'s [30], Wu et al.'s [34], and Baily et al.'s [6] systems actuate the computer monitor to encourage users to sit in an ergonomically correct posture. Sirkin et al. [32] and Wu et al. [34] also explored how to extend this concept to furniture, such as desks, chairs, and ottomans that adapt their configuration to provide the user with an optimal ergonomic setup. Actuated tools 
have also been used to help users avoid mistakes. For example, when carving shapes from a block of material, the actuated mill FreeD [38] prevents users from making a mistake by stopping its spindle if the user's carving would distort the intended shape. Similarly, an actuated knife [37] can prevent users from cutting their fingers by retracting the blade if it gets too close to the fingers. To support users in drawing geometric shapes, such as circles and squares, computer-controlled actuated pens, such as dePENd [36] and IDraw [10], can guide users hands while drawing by moving the pen on the path that achieves the desired drawing output. While these related works demonstrate how actuation can help users to perform motor tasks successfully, our focus is on how to use actuation for the training of motor skills. In particular, we investigate how actuation can increase and decrease task difficulty for training to always maintain difficulty at a level that leads to the largest potential learning benefit.

\subsection{Current Systems for Motor Skills Training}

Researchers have developed several systems to help learners with motor skill training. These systems mainly provide feedback to the learners in the form of vibration, audio, and visual overlays during task execution. Examples include systems for learning skateboarding [25, 26] and snowboarding [24] that provide users with feedback on their foot position and timing of movements by using audio replay or by visualizing users' paths on the floor. Similarly, TapTrain supports roller derby skaters by providing vibrotactile feedback on the execution of crossovers and strides [33]. Systems to support training for golf give users feedback on their body position using a virtual avatar and by replaying the swing sound of the golf club [12, 18, 23]. Similarly, systems to support users in learning tennis provide users with force feedback on the tennis racket to guide them into the correct swing motion [4, 21]. Furthermore, Blank et al.'s system for learning table tennis gives visual feedback to users on the location of the ball hitting the table [7]. Baca et. al's system to support learning to row records ground reaction forces and users' rowing forces and then gives visual feedback to users on force directions [5]. Gu et al.'s system to support learning archery provides vibrotactile feedback to the learner's arms to inform them of unwanted tilting of the bow [13]. Beside sports training, feedback systems also help in learning to play violin [16], to draw calligraphy [15], and to eat with chopsticks [8]. All these systems provide multimodal feedback, such as vibration and audio, to support the training of motor skills. In contrast, we study whether shape adaptation can be used to vary task difficulty to improve training outcomes.

\subsection{Motor Skill Learning by Varying Task Difficulty}

Research in motor skill learning $[28,29]$ has shown that the difficulty level of a learning task plays a major role in determining the extent of learning gains during the learning process [9]. If the task is too easy, learners are underchallenged and if the task is too difficult, learners are overchallenged. In both these cases, learners miss out on the maximum potential learning benefit (see Figure 3). The concept of using appropriate task difficulty for training purposes was first theorized by Kelly [17]. Kelly defined adaptive training as a training in which the problem, the stimulus, or the task is varied as a function of how well the trainee performs. Kelly points out that training with an adaptive difficulty level is efficient because only when the training is at an appropriate level of difficulty, effective learning can occur. He further states that to implement adaptive training, the system requires an adaptive variable, measurement of performance, development of an auto-adaptation logic, calculation of the error, computation of the task difficulty and finally, visualization of the knowledge results. Automatically adapting task difficulty has several benefits in the area of motor skill learning $[1,2,17]$, such as making personalized and optimal learning scalable. However, it is unclear how to automate the adaptation of task difficulty. For instance, while researchers studying motor skill learning have proposed multiple models of evaluating how much a learner learned after training a motor skill [9, 11, 22, 31, 35], no method exists to implement these models for automated training systems. In our work, we demonstrate how to transfer the model of training at the optimal challenge point into an adaptive tool the prototype of an adaptive basketball stand that we built for our user studies. We next describe the design of this prototype, how it measures learners' performance using sensors, and how it adapts task difficulty using integrated actuators.

\section{STUDY PROTOTYPE}

To be able to compare the training with (1) a static, (2) a manually adaptive, and (3) an automatically adaptive tool, we first built a study prototype that works across all three conditions. We first describe the choice of motor skill for the user studies, which determined the choice and design of the study prototype.

\subsection{Choice of Motor skill}

While a wide range of skills can be used to study motor skill learning, such as learning to ride a bike or to skateboard, we chose basketball as an example because the task of shooting balls into the hoop requires only a short amount of time (each shot takes around 3-4 seconds). This allows us to collect more data points in our user studies (220 shots per participant within an hour of study). In addition, a similar motor skill task of throwing balls into a basket on the ground has been previously studied in the field of motor skill learning [19]. Hence, we selected the motor skill of throwing a basketball into a hoop as our study task.

\subsection{Design of the Study Prototype}

Figure $2 \mathrm{a}$ shows the design of the final study prototype of a basketball stand that we used for the three study conditions. The study prototype has an adjustable hoop height and width, i.e., the hoop can widen and tighten and the stand can raise or lower the hoop. It is mounted with: (1) actuators to adjust the hoop height and width (Figure 2b), and (2) sensors to detect when the ball hits the board or goes through the hoop (Figure 2c).

Actuators: To adapt the hoop height, we use a stepper motor (Figure 2b top) integrated with the base of the stand that lowers or raises the hoop. Similarly, to adapt the hoop width, we use a servo motor (Figure $2 \mathrm{~b}$ bottom) integrated with the hoop that increases or decreases the hoop diameter. We chose the height adjustment as one dimension of adaptation because it also exists in manually adjustable commercial basketball setups and thus based our design 

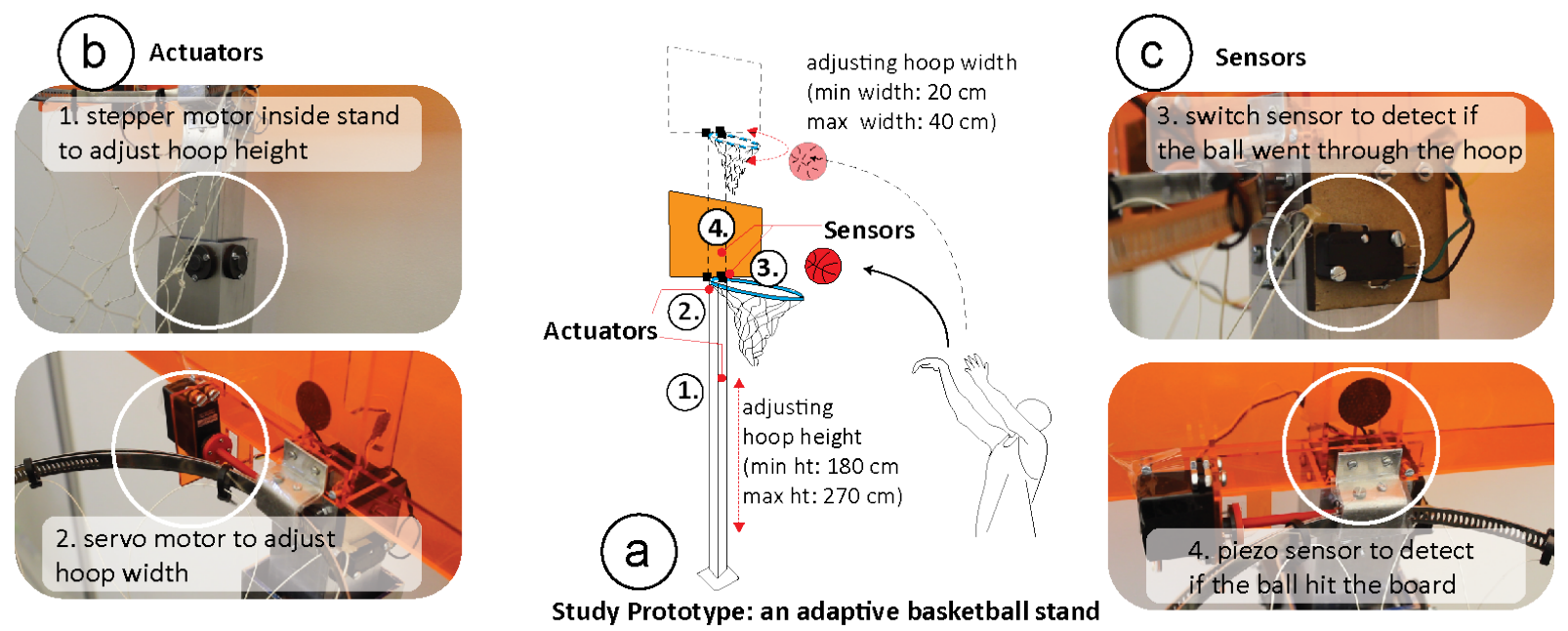

Figure 2: Study Prototype: (a) Our basketball stand with adjustable hoop height (min height: $180 \mathrm{~cm}, \mathbf{m a x}$ height: $270 \mathrm{~cm}$ ) and hoop width (min width: $20 \mathrm{~cm}$, max width: $40 \mathrm{~cm}$ ) is mounted with (b) actuators, i.e. a stepper and a servo motor for adaptation, and (c) sensors, i.e. a switch and a piezo sensor to detect if the ball went through the hoop or hit the board.

on a real-life counterpart. We added a second dimension of adapting the task difficulty by adjusting the hoop width because wider hoops are easier to score with than smaller hoops. While hoop width adjustment is less common in commercial setups, it is used in motor skill training of basketball athletes where hoop variation has shown to improve free-throw performance when correctly adapted by a personal trainer [20]. Using these actuators, the basketball height and width can be adjusted continuously between a height of $180-270 \mathrm{~cm}$ and a hoop diameter of $20-40 \mathrm{~cm}$, respectively. Thus, the lowest difficulty setting is with $180 \mathrm{~cm}$ hoop height and $40 \mathrm{~cm}$ hoop width, and the highest difficulty setting is $270 \mathrm{~cm}$ hoop height and $20 \mathrm{~cm}$ hoop width. For reference, the ball used for the user study had a diameter of $15 \mathrm{~cm}$.

Sensors: We sense the learner's performance by detecting if the ball went through the hoop, hit the board, or completely missed. To sense if the ball went through the hoop, we use a switch sensor attached to the net of the hoop that gets activated when the ball goes through the hoop and pulls on the net (Figure $2 \mathrm{c}$ top). To detect if the ball hit the board, we use a piezo sensor (SEN-10293 from Sparkfun) that detects the noise of the ball hitting the board, which is a reading above 100 units (Figure 2c bottom). If neither the switch is activated nor the piezo reading is above 100 units, we infer that the ball completely missed the basket. While there are many alternative ways to sense a learner's performance in basketball, such as monitoring the trajectory of the ball using a camera, finding the best sensing mechanism was not the focus of our work and we therefore kept the sensor choices at a level sufficient for detecting performance.

Using these sensors, our prototype can sense three performance outcomes, which are in order from most to least successful: basket scored, board hit, and completely missed. We explain more details on monitoring performance in Section '4.3 (Step 1) Monitoring Performance in Practice'.

\subsection{Using the Study Prototype for all three Study Conditions}

Next, we explain the three study conditions and describe how our study prototype supports each of these conditions:

Static Condition: In the static condition, the difficulty level is fixed and thus the prototype does not adapt during training. Therefore, no actuation of the tool or sensing of the user's performance is needed.

Manually Adaptive Condition: In the manually adaptive condition, participants are in control of which difficulty level they want to train on. We provide participants with a keyboard to control the actuators to adjust the hoop width and the hoop height as desired. Thus, this condition requires actuation but does not require sensing of the user's performance.

Auto-Adaptive Condition: For the auto-adaptive condition, our adaptation algorithm controls the difficulty level. To provide the adaptation algorithm with data on the user's performance, the switch and piezo sensors embedded in the study prototype are used to determine if the ball missed the hoop, hit the board, or successfully went through the hoop. Based on the performance data, our algorithm then determines if the actuators need to adapt the tool to the next difficulty level. Thus, both sensors and actuators are required for this condition. In addition, to determine when to use the actuators to adjust the task difficulty, the auto-adaptive condition also requires an algorithm that computes the training task difficulty level. We provide more information on how to determine optimal task difficulty in the next section.

\section{BACKGROUND ON VARYING TASK DIFFICULTY}

As mentioned earlier, one key component of auto-adaptive training tools is to determine when to adapt the tool so that the task difficulty level accommodates the learner's increase in skill level. One way to adapt the difficulty for a learner is by having them train around 
what is called the optimal challenge point [14], which has been proven to lead to higher learning gains. Here, we first describe the relevant concepts and then detail how we translate these concepts into the design of our tool.

\subsection{Task Difficulty}

To understand how the task difficulty during training affects the learning of a motor skill, researchers have defined two types of task difficulties: nominal task difficulty and functional task difficulty [14].

Nominal Task Difficulty (Independent of the Learner): Nominal task difficulty is the level of difficulty of the task independent of the person executing the task and their skill level. For example, in basketball, it is more difficult to score a basket that is mounted at a higher height and has a smaller width, than to score a basket mounted at a lower height and has a larger width, irrespective of the person performing the task. When learners transition from low nominal task difficulty (lowest difficulty setting: minimum height and maximum width) to high nominal task difficulty (highest difficulty setting: maximum height and minimum width), they transition from beginner to expert level. In our study prototype, the lowest nominal task difficulty is at a basket height of $180 \mathrm{~cm}$ and a hoop width of $40 \mathrm{~cm}$, the highest nominal task difficulty is at a basket height of $270 \mathrm{~cm}$ and a hoop width of $20 \mathrm{~cm}$.

Functional Task Difficulty (Dependent on the Skill Level of the Learner): Functional task difficulty refers to how challenging the task is in relation to the person executing the task and their skill level. For instance, when throwing a ball at a high basket, the task will typically be more difficult for a beginner with a low skill level than an expert with a high skill level. Thus, to keep both the beginner and the expert learner at the same functional task difficulty, i.e., challenged in the same way, a beginner would have to train at a low nominal task difficulty (e.g., $180 \mathrm{~cm}$ ), while an expert would have to train at a high nominal task difficulty (e.g., $270 \mathrm{~cm})$. Note that the functional task difficulty for both learners would be the same. As can be seen in Figure 3, functional task difficulty and performance in practice are correlated, for example, a lower functional task difficulty (i.e., an easier task) results in higher performance and vice versa.

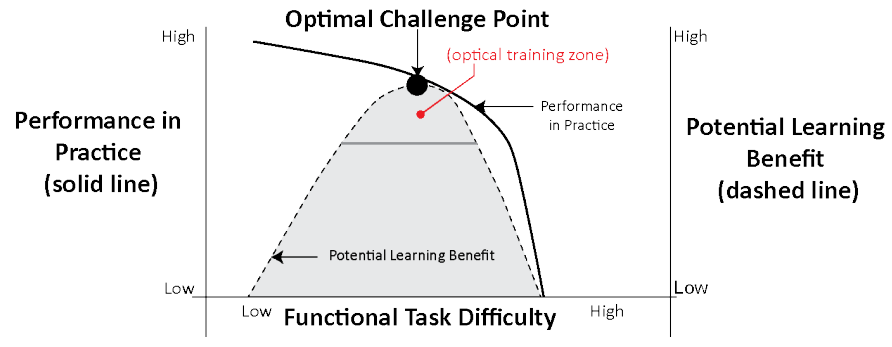

Figure 3: The optimal challenge point is the level of functional task difficulty at which the task is neither too hard nor too easy, which allows for the largest potential learning benefit. Figure adapted from Guadagnoli et al. [14].

\subsection{Optimal Challenge Point}

Research has shown that when the functional task difficulty is at a level that is neither too difficult nor too easy for the learner, it results in the highest potential learning benefit [14]. This level of functional task difficulty is called the optimal challenge point. Figure 3 (adapted from Guadagnoli et al. [14]) illustrates this concept in more detail: when the functional task difficulty is too low, i.e. the task is too easy, the learner's performance in practice is high (e.g., every single basket is scored in basketball), but the learner remains underchallenged and the potential learning benefit is low. Conversely, when the functional task difficulty is too high, i.e., the task is too difficult, the learner's performance in practice is low (e.g., no basket is scored), and the learner is overchallenged and thus this setup also fails to maximize the potential learning benefit. Therefore, to have the highest potential learning benefit, the functional task difficulty needs to be at a medium level, i.e., the task at hand should neither be too difficult nor too easy, which is reflected in a medium performance level in practice (e.g., some baskets are scored but not all). For instance, in our basketball scoring scheme, a learner trains around the optimal challenge point when their average score is around $50 \%-75 \%$ (see Table 1), i.e. some boards and some baskets are hit but not all.

\subsection{Adjusting Task Difficulty to Maintain the Optimal Challenge Point}

We explained above that to allow learners to progress efficiently from a beginner level with low nominal task difficulty to an expert level with high nominal task difficulty, learners need to train at a functional task difficulty set at the optimal challenge point at all times. We now describe how the algorithm accomplishes this using the following series of steps:

Initialize: Lowest Nominal Task Difficulty (Beginner Setting): Our adaptive tools initialize at the beginner level with the lowest nominal task difficulty. In our basketball example, the lowest nominal task difficulty sets the hoop at the minimum hoop height and maximum hoop width.

Step 1. Monitoring Performance in Practice / Functional Task Difficulty: Next, the algorithm monitors the learner's performance by collecting data from the integrated sensors over time. The performance in practice (Figure 3) reflects the level of functional task difficulty that the learner is experiencing. In our work, we calculate the performance using the learner's average score over time. If the functional task difficulty is at a medium level, i.e. neither too difficult nor too easy, the learner trains in the zone around their optimal challenge point, reflected by a medium good score and therefore, our algorithm will keep the nominal task difficulty unchanged, i.e. the physical tool will not adapt. However, because learners' skills tend to improve with practice, which is reflected in an increase in the average score, the task will become too easy as the learner keeps training and the functional task difficulty will become too low, i.e. it will be below the optimal challenge point. In our work, as long as the learner's performance in practice is medium (50\%$75 \%$ success rate on average), our algorithm maintains the current nominal difficulty level (see Table 1). If the score increases to $>75 \%$ success, we adapt to a higher nominal task difficulty level. 


\begin{tabular}{|c|c|c|c|c|}
\hline Average Score $^{*}$ & Task Difficulty & Adaptation & Hoop Width & Hoop Height \\
\hline$<=50 \%$ & high & adapt easier & increase & decrease \\
$>50 \%$ and $<=75 \%$ & optimal challenge point & no change & no change & no change \\
$>75 \%$ and $<=100 \%$ & low & adapt harder & decrease & increase \\
\hline
\end{tabular}

Table 1: Based on the learner's average score (performance), the algorithm detects if the task difficulty is too low, too high, or at the optimal challenge point and then either adapts the hoop width and height to make task more difficult, easier, or keep it same

Step 2. Increase Nominal Task Difficulty by Adapting the Tool: Once the performance in practice is high (functional task difficulty too low), our algorithm increases the nominal task difficulty by adjusting the tool. In our basketball example, the algorithm can increase nominal task difficulty by raising the stand and making the hoop smaller. Since the nominal task difficulty is now higher, the previously too low functional task difficulty will now be back at a higher level around the optimal challenge point, allowing the learner to train again with highest potential learning benefit. For instance, while a learner may have scored enough baskets to accomplish an average score of $>75 \%$ hits with the lower basket height and wider hoop, the new higher basket height and smaller hoop setting may drop their average score back to around $50 \%$.

Repeat step $1+2$ Until Highest Nominal Task Difficulty is reached (Expert Setting): Our algorithm repeats step 1 and step 2, i.e. monitoring performance in practice and increasing the functional task difficulty by adapting the tool, until the highest nominal task difficulty setting is reached. If the functional task difficulty is low at the highest nominal task difficulty, i.e. if the performance is high in the expert setting, the learner has fully mastered the skill.

\subsection{Algorithm for Adaptation}

To implement the above approach for the study prototype of our adaptive basketball stand, we use an algorithm that varies the task difficulty by monitoring the learner's performance using sensors, computes the task difficulty based on the performance data, and then adapts the tool accordingly using actuators. We describe the algorithm we use below:

Step 1. Monitoring Performance: To measure the performance of learners during the training, we use sensor readings to determine if the ball went through the basket, was a board hit or missed completely (as explained in Section 3.2), and then assign performance scores accordingly (see Table 2 ).

\begin{tabular}{|c|c|c|c|}
\hline Switch State & Piezo Reads & Performance & Score \\
\hline activated & - & basket scored & $1 \mathrm{pt}$ \\
not activated & $>=100$ units & board hit, basket missed & $0.5 \mathrm{pt}$ \\
not activated & $<100$ units & board and basket missed & $0 \mathrm{pt}$ \\
\hline
\end{tabular}

Table 2: Mapping of sensor readings to states, scores and performance.

Step 2: Computing Task Difficulty and Adaptation: While adaptation helps to modify the task difficulty to better match the learner's optimal challenge point, too frequent adaptation, i.e., after every attempt, would not adequately reflect a user's performance due to potential outliers, such as a scored basket at random. To better represent the user's current performance level, the algorithm we use averages the performance over a set number of attempts.

To remove the noise in the data, we used two methods, a discrete window for user study 1 and running window approach for user study 2 , both with a window size of 4 attempts. The algorithm then calculated the average score for every attempt using these two approaches. Next, the algorithm then determines if the overall performance is high/low enough to require adaptation of the tool or if the tool should remain in its current difficulty setting (see Table 1). This method allows us to get a better estimate of the user's overall performance and smooth out the noisy data. To ensure that the tool only adapts when the performance is steady, we further compute the derivative of the moving average. When the derivative is 0 , the performance has plateaued, and the adaptation can proceed according to the score of the running average window. We calculated the running average $\left(r_{a v g}\right)$ at $m^{t h}$-attempt with score (s) using a window of $n$-attempts, and the derivative of the moving average at that $m^{t h}$-attempt using the following formula:

$$
\begin{gathered}
\bar{r}_{\mathrm{avg}}=\frac{s_{m}+s_{m-1}+\cdots+s_{m-(n-1)}}{n}=\frac{1}{n} \sum_{i=0}^{n-1} s_{m-i} . \\
d_{m}=r_{\mathrm{avg}(\mathrm{m})}-r_{\mathrm{avg}(\mathrm{m}-1)}
\end{gathered}
$$

Step 3: Amount of Adaptation to Increase Nominal Difficulty: In our prototype and studies, for each adaptation, the algorithm adjusts the basket height by $5 \mathrm{~cm}$ (16 stepper revolutions) and adjusts the hoop diameter by $1-1.5 \mathrm{~cm}$ (8 servo turns). We chose this adaptation based on empirical observation from our pilot studies. While ideally the amount of adaptation would be based on the performance level of the learner (i.e., a higher running average would lead to a larger adaptation amount than a lower running average), we leave this for future work and use a uniform fixed adaptation amount across all performance outcomes.

With the auto-adaptive study prototype and adaptation algorithm ready, we conducted two user studies with 12 participants each to compare training in (1) the static versus auto-adaptive condition and (2) the manually adaptive versus the auto-adaptive condition.

\section{USER STUDY 1: STATIC VS. AUTO-ADAPTIVE TRAINING TOOLS}

In our first user study, we compare the learning gain and training experience of training with a static tool versus an automaticallyadaptive tool. We base our user study on a previous study in motor 

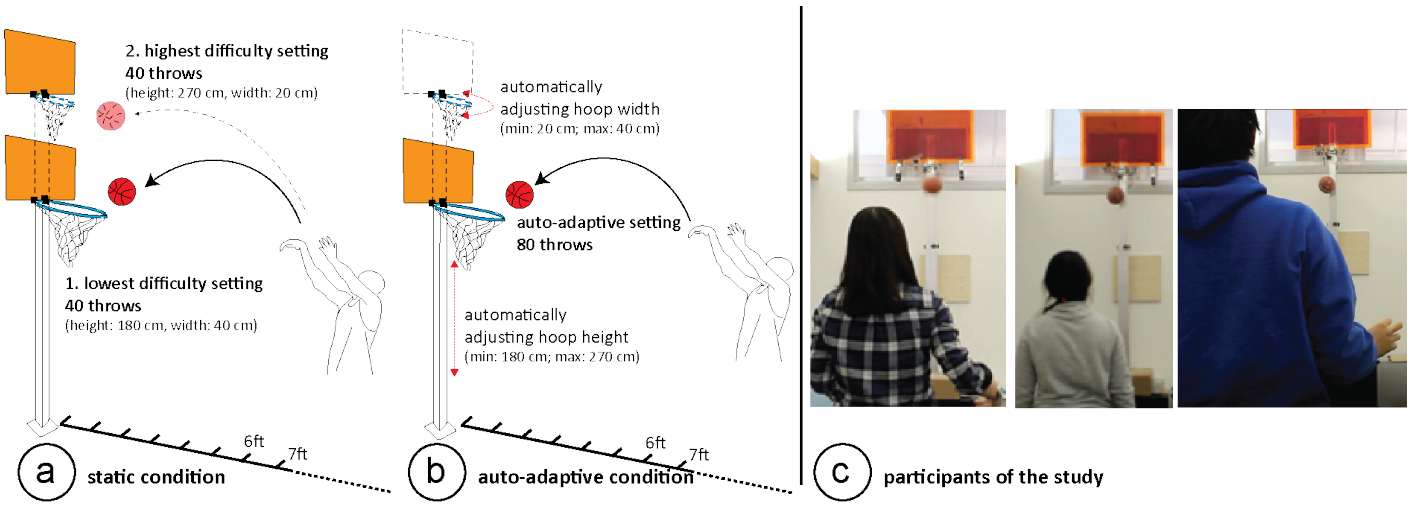

Figure 4: The setup for user-study 1 with two training conditions: (a) static (80 throws: 40 lowest difficulty, 40 highest difficulty setting) and (b) auto-adaptive (80 throws continuously adapting difficulty). (c) The participants of our study seen during training were standing at distances based on a measurement of their initial performance.

skill learning that investigated fixed practice versus varied practice in which a personal trainer adjusted the training, and showed that varied practice leads to a larger learning gain [19].

\subsection{Study Design}

Hypothesis: Our hypothesis was that training with an auto-adaptive tool, which adapts the difficulty level based on the learner's performance, will result in a higher learning gain when compared to a static tool that does not vary the task difficulty based on learner's performance during the training. A higher learning gain will be reflected in a higher performance score after the same amount of training time in each condition. Note that to measure each participant's learning gain for a given condition, we always compared the participant's post-training score with their own pre-training score.

Participants: We recruited 12 participants ( 8 female, 4 male) aged between $18-31$ years $($ mean $=25$, std. deviation $=3.3)$. The mean height of the participants was 5'3" (std. deviation=2.24). 8 participants had last played basketball about 2-5 years ago, while 4 participants said that they last played basketball about 10 years ago. All 12 participants were right-handed. 3 participants had experience with other ball sports, such as volleyball and racquetball.

Setup: Participants were asked to stand at a single location and attempt the throws for the entire study from this position. We used our study prototype without any adaptation in the static condition. For the auto-adaptive condition, we deployed our algorithm on the microcontroller integrated with the basketball stand. We used the algorithm detailed in Section 4.4. and used a window size of 4 attempts (i.e., 4 throws at the basket) based on empirical observations from our pilot study. Using a smaller window than 4 would result in a constant adaptation of the tool because of the strong effect of the outliers (e.g., hitting the basket by chance). In contrast, a larger window may result in too slow adaptation (i.e., learners may move away from the optimal challenge point before the moving average plateaus).
Conditions: The study followed a within-subjects design. We used randomized order to avoid order effects for training the participants on the following two conditions:

Static training: In the static training condition, participants attempted half (40) the throws in the lowest difficulty setting (i.e., largest hoop, lowest basket height) and the second half (40) of throws in the highest difficulty setting (i.e., the smallest hoop and the highest basket height). This condition replicates the use of most training aids today that are not adaptable, i.e. most training aids only come in beginner/expert versions (e.g., either use the wide beginner basketball hoop or the regular hoop, but nothing in-between).

Auto-adaptive training: In the auto-adaptive training condition, participants started by throwing balls in the lowest difficulty setting (lowest basket height and largest hoop size) and gradually progressed towards the harder settings using the adaptation algorithm shown in Table 1 as they performed a total of 80 throws.

Study Procedure: The study procedure for each participant followed the six steps listed below:

Pre-Study (Calibration of Distance to Basket): When piloting the study, we noticed that a tall participant would find the highest difficult level of the study too easy and a short person would find the lowest difficulty level too difficult. To compensate for these differences we calibrated the distance from the basket for each participant (Figure 4). To calibrate the distance, each participant attempted 4 throws from $6 \mathrm{ft}$ distance. If the score was $50 \%$ or less, the participant continued the rest of the study with that distance. If the score was higher, the participant moved $1 \mathrm{ft}$ away and repeated the process until the score was $50 \%$ or less. The mean distance at which the participants stood for the study was $9.8 \mathrm{ft}$ (std. deviation $=2.07 \mathrm{ft}$ ).

Study Steps: In total, each participant attempted 220 throws over the 5 phases of the study (see Table 3). For training condition \#1, the participants were randomly assigned either static training or auto-adaptive training first, and then the respective other training in condition \#2. In all phases, we instructed participants to score as many points as possible. To avoid fatigue, participants were asked 
to take a 5 minute break after each of the above steps. In total, the study took one hour per participant, including the pre-study and post-study questionnaire. Because the study followed a withinsubjects design with randomized order, 6 participants trained on the adaptive condition first and the other 6 participants trained on the static condition first.

\begin{tabular}{|c|c|}
\hline Study Procedure Steps & Throws \\
\hline pre-training skill assessment test & 20 \\
training condition \#1 & 80 \\
post-training test \#1 & 20 \\
training condition \#2 & 80 \\
post-training test \#2 & 20 \\
\hline total attempts & 220 \\
\hline
\end{tabular}

Table 3: Every participant attempted 220 throws during the study.

Measuring Learning Gain: To measure the learning gains for the first condition participants trained in, we compared the participant's score in the pre-training skill assessment test and their score in post-training test \#1. To measure the learning gains of the second condition participants trained in, we compared their score in post-training test \#1 and their score in post-training test \#2. The learning gains were measured by calculating the differences in the respective test scores.

\subsection{Study Results: Average Learning Gain}

We calculated the average learning gains for both the conditions and found that the training in the auto-adaptive condition led to a $20 \%$ increase in the average performance score compared to the static training condition. As seen in Figure 5, participants scored a total of 4 points more on average during their 20 attempted throws in the post auto-adaptive training test. (With maximum 1pt per attempt, the maximum possible score was 20 points in each post-training test). ANOVA analysis showed that the increase in the learning gains in the auto-adaptive condition was statistically significant $\left(F_{1,11}=1.856, p<0.05\right)$.

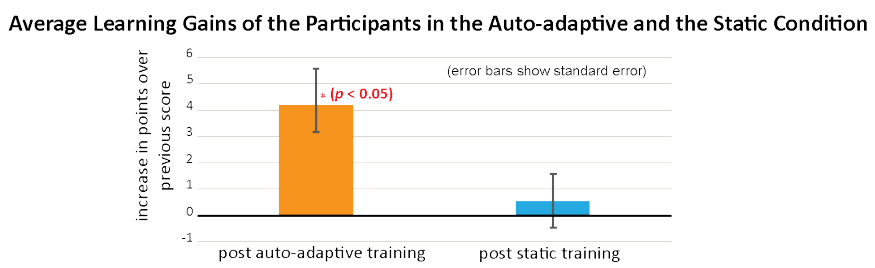

Figure 5: User-study 1: Average learning gain post adaptive training and post non-adaptive training. The average learning gain post adaptive training is significantly higher than performance post static training $\left(F_{1,11}=1.856, p<0.05\right)$.

Difficulty and performance over time: Plotting the average score of participants (Figure 6) shows that during the auto-adaptive training, the average performance score of participants was between $40 \%$ and $75 \%$, which is within the range of the optimal challenge point. In contrast, in the static condition the average performance score of participants dropped below $40 \%$ demonstrating that the setup was too difficult and participants trained on a difficulty level that was further away from the optimal challenge point and thus provided less optimal learning gains.

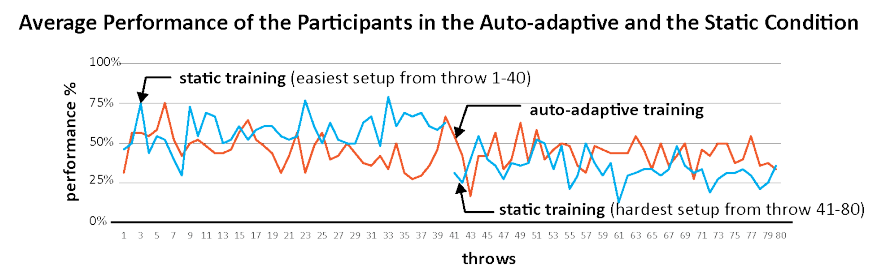

Figure 6: Average performance of the 12 participants during the static training condition and the auto-adaptive training condition over 80 throws.

\subsection{Qualitative feedback}

Apart from quantitative results, we also collected qualitative feedback on the experience of training with an auto-adaptive tool. When participants were asked if they would rather retrain with the autoadaptive or the static condition, 8 out of 12 participants preferred the auto-adaptive condition. On a 5-point Likert scale, the median preference was 4 (std. deviation $=0.86$ ) for the auto-adaptive vs. 3 (std. deviation $=0.90$ ) for the static condition. A KruskalWallis test showed the difference was not statistically significant.

Participants' Preferred Condition for Motor-skill Training - Likert Scale

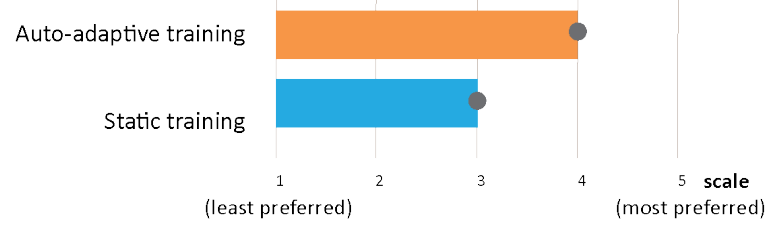

Figure 7: Bar chart showing median and mod of preference (on a scale of 1-5) of the participants for training in each condition when asked to rate their preference to retrain on the auto-adaptive and the static condition.

When asked about the reasons for their preferences, participants stated the following:

Seeing the physical tool adapt: 'The adaptive training made me reflect upon what I'm doing wrong by physically changing the height and hoop size and made me want to change my throwing style faster.' - (p3), 'Adaptive training makes each stage of the training experience more rewarding, so it helped me focus better.' - (p11), 'Adaptive mode let me see gradual progress, while non-adaptive mode just felt like either shots that were too easy or an exercise in futility.' - (p8), 'Changes with the hoop was cool [...] like having a personal trainer motivate me [...] when it was getting tighter, I would think I'm doing good, when it got loose I would think I should do better.'- (p7).

Having adaptive difficulty levels: 'The adaptive mode felt more useful in slowly adjusting to let me get better at harder and harder shots' - (p8). 'it's more natural! [...] it matches learning 
process better' - (p7). However, one participant also mentioned 'I prefer to be challenged beyond my current capability and learn that way. - (p10)

Adaptive training versus static training: Some participants stated that they felt they might perform better by only practicing on the most difficult setting. 'I feel like I get better if I just practice with hard level and nothing else.' - (p5), '[while] adaptive training is more interesting, non-adaptive training helps me improve specific movements.' - (p4) These comments are particularly noteworthy because it is common for learners to assume that training in the most difficult setting or a single setting enables faster skill acquisition. However, studies [19] show that when learners train with variation in practice, they perform better than when training on a static setting and our results align with the outcomes of these studies. In the Section for 'optimal challenge point', we described in detail why training on a setting that is either too difficult or too easy is non-optimal because it misses the maximum learning potential for the learners.

To summarize the qualitative feedback, we discovered that learners find it motivating to see their progress through tool adaptation without having to focus on constantly assessing their own performance. While results show that the participants performed better after auto-adaptive training, we also observed that learners felt that they would have performed better if they were allowed to choose the difficulty level for their training themselves. To test if this would indeed lead to an improvement over auto-adaptive training, we ran a second study to compare manually adaptive versus auto-adaptive training.

\section{USER STUDY 2: MANUALLY ADAPTIVE VS. AUTO-ADAPTIVE TRAINING TOOLS}

For our second study, we investigated if automatically adapting the training tools also leads to higher learning gains when compared to manually adaptive training tools, i.e. when the learners have the choice to adjust the difficulty level according to their preference.

\subsection{Study Design}

Hypothesis: We hypothesized that the training with an automaticallyadaptive training tool will result in a higher learning gain when compared to a manually adaptive training tool. This will be reflected in a higher performance score after the same amount of training time.

Participants: We recruited 12 participants (8 females) aged between $19-28$ years $($ mean $=21$, std. deviation $=2.62)$. The mean height of the participants was 5'6" (std. deviation $=4.3$ ). The frequency of playing basketball was: once a week $=1$ no., once a month $=1$ no., $2-5$ years ago $=5$ nos., 10 years ago $=4$ nos., never $=1$ nos. 11 participants were right handed and 1 participant was left handed. 2 participants had experience with other sports such as football and water-polo.

Setup: We used the same room setup and basketball prototype as in the prior user study. For the automatic adaptation, we deployed the adaptation algorithm on the micro-controller integrated with the basketball stand and used the auto-adaptation algorithm described in Section 4.4. with a window size of 4 attempts. In the manually adaptive condition, participants controlled the adaptation, i.e. they could decide which difficulty level to train on and for how long to train in the setting.

Conditions: As in the previous study, this study followed a within-subjects design and randomized order to avoid order effects for training the participants on the following two conditions:

Manually Adaptive training: For the manual adaptation, we added a keyboard interface, which participants used to adjust the basket height (arrow up/down) and hoop diameter (arrow left/right) according to their preference.

Auto-adaptive training: In the auto-adaptive training condition, our adaptation algorithm automatically adjusted the tool during the training, as described in the Section 'Setup' above.

Study Procedure: The study procedure was the same as in the first study (refer to Section 5.1), i.e. we started with a pre-study calibration of the distance to the basket, followed by 220 throws (pre-training skill assessment: 20 throws, training condition 1: 80 throws, post-training 1 test: 20 throws, training condition 2: 80 throws, and post-training 2 test: 20 throws) as shown in Table 3.

Measuring Learning Gain: We measured learning gain in the same way as in the first study (refer to Section 5.1).

\subsection{Study Results: Average Learning Gain}

We calculated the average learning gains for both the conditions and found that the training in the auto-adaptive condition led to a $25 \%$ increase in the average performance score compared to the manually adaptive condition. As can be seen in Figure 8, participants scored 5 points higher on average during their 20 attempted throws in the post auto-adaptive training test (each basket hit = 1pt). ANOVA analysis showed that the increase in the learning gains in the auto-adaptive condition was statistically significant $\left(F_{1,11}=2.386, p<0.05\right)$.

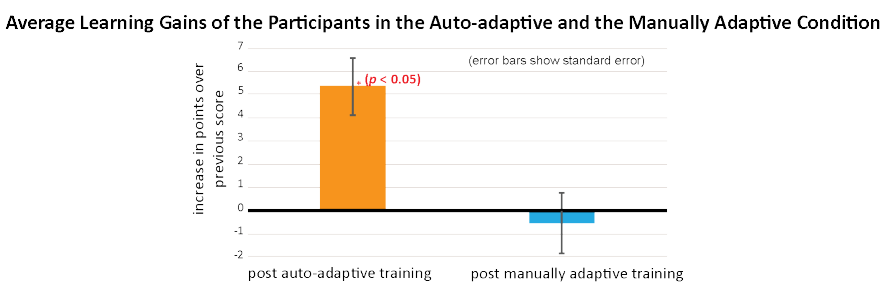

Figure 8: User study \#2: Average learning gain of post autoadaptive and manually adaptive training. The average learning gain in performance post auto-adaptive training is significantly higher than the performance post manually adaptive training $\left(F_{1,11}=2.386, p<0.05\right)$.

Maintaining Functional Task Difficulty Around the Optimal Challenge Point: It was evident from the results of the manually adaptive condition that participants failed to determine the difficulty level that offers the largest learning gain when left to configure the hoop height and width themselves. Figure 9 shows 
the scores and adaptation frequency of one of the participants (p2) for both the manually and auto-adaptive conditions. As can be seen in Figure 9a, in the manually adaptive condition, p2 increased the difficulty too quickly, i.e. after every successful score $\mathrm{p} 2$ raised the hoop to a larger height and decreased the hoop width. As a result, p2 was over-challenged around throw number 30 and missed more often (see low performance scores from attempt 30 onward). In contrast, in the auto-adaptive condition, 22 progressed steadily as seen by the regularity in the frequency of adaptation in Figure 9b. P2 also scored more consistently during the auto-adaptive condition, i.e. during the entire duration of the training the success score was approximately the same.

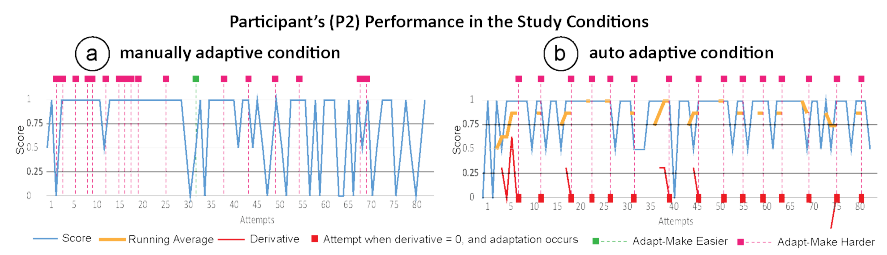

Figure 9: Performance score and adaptation frequency of p2. (a) In the manually adaptive condition, p2 increased the difficulty too quickly, i.e. after every successful attempt, which soon led to $\mathrm{p} 2$ to being overchallenged as reflected by the low scores starting around throw 30. (b) In contrast, in the automatically-adaptive mode a uniform difficulty level was maintained throughout the training as reflected by the steady scoring.

\subsection{Qualitative Feedback}

When participants were asked if they would rather retrain with the automatically-adaptive or manually adaptive condition, 10 out 12 participants preferred the automatically-adaptive setup. On a 5-point Likert scale, the median preference was 5 (std. deviation $=0.76$ ) for auto-adaptive vs. 3 (std. deviation $=0.87$ ) for manually adaptive, and the KruskalWallis test showed the difference was statistically significant $(\mathrm{p}<0.05)$. When asked to highlight the benefits and drawbacks of each condition, participants responded as follows:

Self-evaluation: 'There were times during the manually adaptive process where I was unsure what the optimal path to training would be.' - (p5), 'Somehow I trust auto-adaptive training more than myself and maybe I should (as suggested by scores after each type of training received).' - (p2), 'Auto-adaptive is nicer than manually adaptive when the difficulty is increased because it is difficult for me to gauge how much I should increase the difficulty.' - (p7). 'Manually adaptive mode was a little hard to adjust because I didn't know what height to begin at.' - (p4).

Learning of skill, ease and efficiency in training: 'The autoadaptive was easier to use; I didn't have to think about if I set it too easy or too hard.' - (p3). 'Manually adaptive training took longer/hard to judge which increments to practice/alter. It seemed the auto-adaptive training was more efficient.' - (p10).
Participants' Preferred Condition for Motor-skill Training - Likert Scale

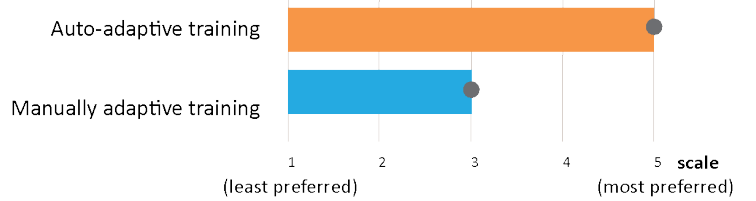

Figure 10: Bar chart showing median and mod of preference (on a scale of 1-5) of the participants for training on each category when asked to rate their preference to retrain on the auto-adaptive and the manually adaptive condition from user study 2 .

Motivation: '[auto-adaptive:] I felt myself getting better and think this would have been really fun to have as a kid.' - (p8), 'I feel that I am doing better every time it adapts to a harder setting.' - (p12), 'I have freedom to grow and make mistakes. No one is watching me (outside of lab setting) so it is more comfortable [than having a personal trainer].' - (p1)

\subsection{Observations on Adaptation Strategies in Manually Adaptive Mode}

During the study, we observed that participants who were assigned to the automatically adaptive condition first used a similar strategy as our algorithm for their manually adaptive condition, i.e. they used similar increments and timings for adaptation in the manually adaptive mode. In contrast, participants who trained on the manually adaptive condition first were fixated on the least difficult or the most difficult setup. For instance, we observed that two participants (p1, p11) chose to train only on the most difficult setup in the manually adaptive mode as they assumed training on the same setup as the test setup would lead to better performance. However, their test score was worse than their pre-training score. This further emphasizes the importance of varied practice over training on a specific goal task [19].

In summary, our results confirm that maintaining functional task difficulty around the level of the optimal challenge point is beneficial in the learning of motor skills and demonstrate that this process can be automated through an adaptation algorithm and the supporting adaptation hardware (sensors and actuators integrated with the tool). Finally, feedback from participants showed that training on the automatically adaptive mode also provides additional motivation and is engaging and more enjoyable. Thus, with automatically adaptive tools, learners can receive personal training based on their own skill level and train at their own pace, making such personalized learning accessible beyond those that have access to a personal trainer.

\section{DISCUSSION}

We showed that automatically adapting physical tools that vary task difficult are effective for motor skill learning as they maintain the training at the optimal challenge point for the learners. We now discuss benefits and limitations of our work. 
Scaling Personalized Training of Motor Skills: As mentioned at the beginning of this paper, one of the main motivations for building automatically-adaptive tools for motor skills learning is that they allow to scale-up personalized learning of motor skills and make it available to a larger audience that does not have access to personal trainers. While we did not compare our automaticallyadaptive tool to learning with a personal trainer, we did provide evidence that our approach leads to significantly higher learning gains when compared to conventional training tools, such as static and manually adaptive tools, that are currently used by learners who do not have access to a personal trainer.

Extending to Other Motor Skills and Tools: While in this paper, we were only able to study one particular adaptive tool, our work can be replicated to study additional tools in the future. For instance, the adaptation algorithm generalizes across different types of tools as long as they have sensors to measure a user's performance and actuators to adapt the difficulty level of the tool. Similarly, while the hardware of our tool, i.e. the particular sensors and actuators, are necessarily specific, we outlined the general requirements that study prototypes need to fulfill to work across all three conditions. We hope that our work can lay the foundation for the study of a variety of adaptive training tools, which will allow researchers to gather more evidence of the effectiveness of adaptive training tools for motor skill training.

Longitudinal Studies with Diverse Participants: While the insights from our study are promising, more longitudinal studies with more varied populations are needed before we can draw conclusions on long-term learning gains. Our study focused on only one adaptive training tool and more studies are needed to confirm the results across a broader set of different tools in different application domains. Furthermore, additional studies need to be conducted to study specific aspects of the skills, such as variation in throwing angle and throwing distance.

Improvements for the Auto-Adaptation Algorithm: In our work, we discussed the discrete vs. running average approach and an additional derivative check to calculate the user's performance over time which we then used to determine when to adjust the difficulty level for training. However, several other approaches can be used to determine when to adapt the tool. For instance, by adding a hysteresis value to the algorithm, we can further enhance our algorithm to only adapt the tool when the performance is at a certain value for a long period of time. Such a hysteresis value can prevent unwanted frequent switching between states when performance fluctuates around the optimal challenge point. In addition, our algorithm currently uses a fixed increase for the adaptation. For future work, we plan to determine not only when to adapt the tool but also how much to adapt it based on the user's performance.

\section{CONCLUSION}

In this paper, we showed that automatically adaptive tools that vary task difficulty based on a learner's performance, can indeed help in motor skill training. Using our study prototype and the adaptation approach of the optimal challenge point, we demonstrated that training in an auto-adaptive condition leads to higher learning gains when compared to training in a static or manually adaptive condition. We showed that the experience of training in the auto-adaptive condition is also more enjoyable for learners since it removes the decision making process around which difficulty level to train on and provides feedback to the learner in the form of the shape-adaptation of the tool. For future research, we plan to build a toolkit that helps designers build their own adaptive training tools. Lastly, we also plan to combine the shape-adaptation with multi-modal feedback and study the combined learning effect.

\section{ACKNOWLEDGMENTS}

This work is supported by MIT Learning Initiative and the National Science Foundation under Grant No. 1844406.

\section{REFERENCES}

[1] ZA Abbas and Jamie S North. 2018. Good-vs. poor-trial feedback in motor learning: The role of self-efficacy and intrinsic motivation across levels of task difficulty. Fournal of Learning and Instruction 55 (2018), 105-112. https://doi.org/ 10.1016/j.learninstruc.2017.09.009

[2] Jack A Adams. 1971. A closed-loop theory of motor learning. Fournal of motor behavior 3, 2 (1971), 111-150. https://doi.org/10.1080/00222895.1971.10734898

[3] Jason Alexander, Anne Roudaut, Jürgen Steimle, Kasper Hornbæk, Miguel Bruns Alonso, Sean Follmer, and Timothy Merritt. 2018. Grand challenges in shape-changing interface research. In Proceedings of the 2018 CHI Conference on Human Factors in Computing Systems (CHI'18). 1-14. https://doi.org/10.1145/ 3173574.3173873

[4] Arnold Baca and Philipp Kornfeind. 2004. Real time detection of impact positions in table tennis. Fournal of The Engineering of Sport 5 (2004), 508-514.

[5] Arnold Baca and Philipp Kornfeind. 2006. Rapid feedback systems for elite sports training. Fournal of IEEE Pervasive Computing 5, 4 (2006), 70-76. https: //doi.org/10.1109/MPRV.2006.82

[6] Gilles Bailly, Sidharth Sahdev, Sylvain Malacria, and Thomas Pietrzak. 2016. LivingDesktop: Augmenting desktop workstation with actuated devices. In Proceedings of the 2016 CHI Conference on Human Factors in Computing Systems (CHI'16). 5298-5310. https://doi.org/10.1145/2858036.2858208

[7] Peter Blank, Thomas Kautz, and Bjoern M Eskofier. 2016. Ball impact localization on table tennis rackets using piezo-electric sensors. In Proceedings of the 2016 ACM International Symposium on Wearable Computers. 72-79. https://doi.org/10. 1145/2971763.2971778

[8] Foong-Yi Chia and Daniel Saakes. 2014. Interactive training chopsticks to improve fine motor skills. In Proceedings of the 11th Conference on Advances in Computer Entertainment Technology (ACE'14). 1-4. https://doi.org/10.1145/2663806.2663816

[9] William H Edwards. 2010. Motor learning and control: From theory to practice. Cengage Learning.

[10] Piyum Fernando, Roshan Lalintha Peiris, and Suranga Nanayakkara. 2014. IDraw: towards a freehand drawing assistant. In Proceedings of the 26th Australian Computer-Human Interaction Conference on Designing Futures: the Future of Design. 208-211. https://doi.org/10.1145/2686612.2686644

[11] Paul M Fitts. 1954. The information capacity of the human motor system in controlling the amplitude of movement. Fournal of experimental psychology 47, 6 (1954), 381. https://doi.org/10.1037/h0055392

[12] Hassan Ghasemzadeh, Vitali Loseu, Eric Guenterberg, and Roozbeh Jafari. 2009. Sport training using body sensor networks: A statistical approach to measure wrist rotation for golf swing. In Proceedings of the Fourth International Conference on Body Area Networks. 1-8. https://doi.org/10.4108/ICST.BODYNETS2009.6035

[13] Heng Gu, Kai Kunze, Masashi Takatani, and Kouta Minamizawa. 2015. Towards performance feedback through tactile displays to improve learning archery. In $\mathrm{Ad}$ junct Proceedings of the 2015 ACM International foint Conference on Pervasive and Ubiquitous Computing and Proceedings of the 2015 ACM International Symposium on Wearable Computers. 141-144. https://doi.org/10.1145/2800835.2800893

[14] Mark A Guadagnoli and Timothy D Lee. 2004. Challenge point: a framework for conceptualizing the effects of various practice conditions in motor learning. fournal of motor behavior 36, 2 (2004), 212-224. https://doi.org/10.3200/JMBR.36. 2.212-224

[15] Naoyuki Houri, Hiroyuki Arita, and Yutaka Sakaguchi. 2011. Audiolizing body movement: its concept and application to motor skill learning. In Proceedings of the 2nd Augmented Human International Conference. 1-4. https://doi.org/10. $1145 / 1959826.1959839$

[16] Rose Johnson, Janet van der Linden, and Yvonne Rogers. 2011. Real-time feedback for learning the violin. In Proceedings of HCI 2011 The 25th BCS Conference on Human Computer Interaction 25. 535-538. https://doi.org/10.14236/ewic/HCI2011. 3

[17] Charles R Kelley. 1969. What is adaptive training? Human Factors 11, 6 (1969), 547-556. https://doi.org/10.1177/001872086901100602 
[18] Philip Kelly, Aoife Healy, Kieran Moran, and Noel E O'Connor. 2010. A virtual coaching environment for improving golf swing technique. In Proceedings of the 2010 ACM workshop on Surreal media and virtual cloning. 51-56. https: //doi.org/10.1145/1878083.1878098

[19] Robert Kerr and Bernard Booth. 1978. Specific and varied practice of motor skill. fournal of Perceptual and motor skills 46, 2 (1978), 395-401. https://doi.org/10. $1177 / 003151257804600201$

[20] Riadh Khlifa, Ridha Aouadi, Roy Shephard, Mohamed Souhaiel Chelly, Souhail Hermassi, and Tim J Gabbett. 2013. Effects of a shoot training programme with a reduced hoop diameter rim on free-throw performance and kinematics in young basketball players. Fournal of Sports Sciences 31, 5 (2013), 497-504 https://doi.org/10.1080/02640414.2012.736634

[21] Laura Marchal-Crespo, Mark van Raai, Georg Rauter, Peter Wolf, and Robert Riener. 2013. The effect of haptic guidance and visual feedback on learning a complex tennis task. Fournal of Experimental brain research 231, 3 (2013), 277-291. https://doi.org/10.1007/s00221-013-3690-2

[22] Karl M Newell, Yeou-Teh Liu, and Gottfried Mayer-Kress. 2001. Time scales in motor learning and development. fournal of Psychological review 108, 1 (2001), 57. https://doi.org/10.1037/0033-295X.108.1.57

[23] Stina Nylander, Alex Kent, and Jakob Tholander. 2014. SwingSound-Experiencing the Golf Swing through Sound. (2014), 443-446. https://doi.org/10.1145/2559206. 2574789

[24] Hyung Kun Park and Woohun Lee. 2016. Motion Echo Snowboard: Enhancing Body Movement Perception in Sport via Visually Augmented Feedback. In Proceedings of the 2016 ACM Conference on Designing Interactive Systems (DIS'16). 192-203. https://doi.org/10.1145/2901790.2901797

[25] Hyung Kun Park, HyeonBeom Yi, and Woohun Lee. 2017. Recording and sharing non-visible information on body movement while skateboarding. In Proceedings of the 2017 CHI Conference on Human Factors in Computing Systems (CHI'17). 2488-2492. https://doi.org/10.1145/3025453.3025476

[26] Sebastiaan Pijnappel and Florian 'Floyd' Mueller. 2014. Designing interactive technology for skateboarding. In Proceedings of the 8th International Conference on Tangible, Embedded and Embodied Interaction (TEI'14). 141-148. https://doi org/10.1145/2540930.2540950

[27] Majken K. Rasmussen, Esben W. Pedersen, and Marianne G. Petersen. 2012 Proceedings of Shape-Changing Interfaces: A Review of the Design Space and Open Research Questions. 735-744. https://doi.org/10.1145/2207676.2207781

[28] Alan W Salmoni, Richard A Schmidt, and Charles B Walter. 1984. Knowledge of results and motor learning: a review and critical reappraisal. fournal of
Psychological bulletin 95, 3 (1984), 355. https://doi.org/10.1037/0033-2909.95.3.355 [29] Richard A Schmidt. 1975. A schema theory of discrete motor skill learning. fournal of Psychological review 82, 4 (1975), 225. https://doi.org/10.1037/H0076770

[30] Joongi Shin, Woohyeok Choi, Uichin Lee, and Daniel Saakes. 2018. Actuating a Monitor for Posture Changes. In Extended Abstracts of the 2018 CHI Conference on Human Factors in Computing Systems (CHI'18). 1-6. https://doi.org/10.1145/ 3170427.3188562

[31] Isabelle M Shuggi, Hyuk Oh, Patricia A Shewokis, and Rodolphe J Gentili. 2017. Mental workload and motor performance dynamics during practice of reaching movements under various levels of task difficulty. Fournal of Neuroscience 360 (2017), 166-179. https://doi.org/10.1016/j.neuroscience.2017.07.048

[32] David Sirkin, Brian Mok, Stephen Yang, and Wendy Ju. 2015. Mechanical ottoman: how robotic furniture offers and withdraws support. In Proceedings of the Tenth Annual ACM/IEEE International Conference on Human-Robot Interaction. 11-18. https://doi.org/10.1145/2696454.2696461

[33] Craig D Stewart, Penny Traitor, and Vicki L Hanson. 2014. I'd Tap That! providing real time feedback on roller derby skills. In CHI'14 Extended Abstracts on Human Factors in Computing Systems (CHI'14). 2221-2226. https://doi.org/10.1145/ 2559206.2581310

[34] Yu-Chian Wu, Te-Yen Wu, Paul Taele, Bryan Wang, Jun-You Liu, Pin-sung $\mathrm{Ku}$, Po-En Lai, and Mike Y Chen. 2018. Activeergo: Automatic and personalized ergonomics using self-actuating furniture. In Proceedings of the 2018 CHI Conference on Human Factors in Computing Systems (CHI'18). 1-8. https: //doi.org/10.1145/3173574.3174132

[35] Gabriele Wulf, Charles Shea, and Rebecca Lewthwaite. 2010. Motor skill learning and performance: a review of influential factors. Fournal of Medical education 44, 1 (2010), 75-84. https://doi.org/10.1111/j.1365-2923.2009.03421.x

[36] Junichi Yamaoka and Yasuaki Kakehi. 2013. dePENd: augmented handwriting system using ferromagnetism of a ballpoint pen. In Proceedings of the 26th annual ACM symposium on User interface software and technology (UIST'13). 203-210. https://doi.org/10.1145/2501988.2502017

[37] Amit Zoran, Nan-Wei Gong, Roy Shilkrot, Shuo Yan, and Pattie Maes. 2015. Cutting Edge Vision: Metal Embedded Optics for Smart Knives. In Proceedings of the 33rd Annual ACM Conference Extended Abstracts on Human Factors in Computing Systems. 1223-1228. https://doi.org/10.1145/2702613.2732495

[38] Amit Zoran and Joseph A Paradiso. 2013. FreeD: a freehand digital sculpting tool. In Proceedings of the SIGCHI Conference on Human Factors in Computing Systems. 2613-2616. https://doi.org/10.1145/2470654.2481361 